\title{
Frequency dependence and intensity fluctuations due to shallow water internal waves
}

\author{
Mohsen Badiey ${ }^{\mathrm{a})}$ \\ College of Marine and Earth Studies, University of Delaware, Newark, Delaware 19716 \\ Boris G. Katsnelson ${ }^{\text {b) }}$ \\ Voronezh State University, Universitetskaya Sq.1, Voronezh 394006, Russia \\ James F. Lynch ${ }^{\mathrm{c})}$ \\ Woods Hole Oceanographic Institution, Woods Hole, Massachusetts 02543
}

Serguey Pereselkov

Voronezh State University, Universitetskaya Sq. 1, Voronezh 394006, Russia

(Received 27 July 2006; revised 16 February 2007; accepted 8 March 2007)

\begin{abstract}
A theory and experimental results for sound propagation through an anisotropic shallow water environment are presented to examine the frequency dependence of the scintillation index in the presence of internal waves. The theory of horizontal rays and vertical modes is used to establish the azimutal and frequency behavior of the sound intensity fluctuations, specifically for shallow water broadband acoustic signals propagating through internal waves. This theory is then used to examine the frequency dependent, anisotropic acoustic field measured during the SWARM'95 experiment. The frequency dependent modal scintillation index is described for the frequency range of $30-200 \mathrm{~Hz}$ on the New Jersey continental shelf. () 2007 Acoustical Society of America.
\end{abstract}

[DOI: $10.1121 / 1.2722052$ ]

PACS number(s): 43.30.Bp, 43.30.Dr, 43.30.Es, 43.30.Zk [WMC] Pages: 747-760

\section{INTRODUCTION}

The study of broadband acoustic wave propagation in shallow water in the presence of inhomogeneities has attracted increasing attention in recent years, with one of the more important sub-topics being the study of the intensity fluctuations of the acoustic field due to nonlinear internal waves. ${ }^{1-6}$ In particular, the frequency dependence of the variability of sound propagation in shallow water is of interest and is closely related to the spatial and temporal variability of physical oceanographic entities such as internal waves. We have recently presented studies ${ }^{6}$ showing the frequency dependence of the refraction of horizontal rays, that correspond to specific acoustic normal modes, using the Weinberg-Burridge formalism. ${ }^{7}$ In our previous theoretical $^{8-11}$ and experimental ${ }^{2}$ papers, we showed that for an acoustic track placed approximately parallel to the wave fronts of internal solitary waves (ISWs), significant horizontal refraction takes place, leading to rather remarkable intensity fluctuations due to the "focusing and defocusing" of the horizontal rays (HRs) during the passage of the internal wave solitons.

In the present paper, we consider this frequency dependence in more detail, both theoretically and using further experimental data from the SWARM'95 experiment. ${ }^{3}$ In particular, we consider the frequency dependence of the fluctua-

\footnotetext{
a) Author to whom correspondence should be addressed. Electronic mail: badiey@udel.edu

${ }^{\text {b) }}$ Electronic mail: katz@ phys.vsu.ru

${ }^{c)}$ Electronic mail: jlynch@whoi.edu
}

tions of the modal intensity as a function of geotime. It was noted $^{6}$ that the shape of the amplitude dependence on frequency for different modes repeats the shape of the frequency dependence of the refraction index in the horizontal plane for a given mode number. Because this behavior is intuitively clear, our previous paper did not present a formal explanation of this phenomenon. In this paper we give an explanation in ray language, and point explicitly to the areas in the horizontal plane where we can observe such behavior. Additionally, we compare theory with experimental results obtained in the SWARM'95 experiment for different positions of the sources, frequency bands, and time periods.

We start by considering the properties of sound radiated by a point source in the horizontal plane, using the Weinberg-Burridge "horizontal rays and vertical modes" formalism. ${ }^{8}$ To do this, we construct a diagram (Fig. 1), where, in the horizontal $(X Y)$ plane, the wave fronts of the internal solitary waves (ISWs) are placed parallel to the $X$ axis, so that the ISW train propagates along the $Y$ axis. We place the acoustic source at the origin. We will be interested in the dependence of the propagation on the horizontal angle $\chi$ between the $X$ axis and the direction of the acoustic track. We then divide the horizontal plane into four quadrants. Because we are only interested in forward scattering, and due to the left-right symmetry of the forward scattering, we need consider only the first quadrant as shown in Fig. 1. The sectors that we divide the first quadrant into are denoted by different radial lines, defining an angular region between the direction of the internal wave front and a given acoustic track. They are also marked by different acronyms. We now explain the meaning of these sectors and their acronyms. 


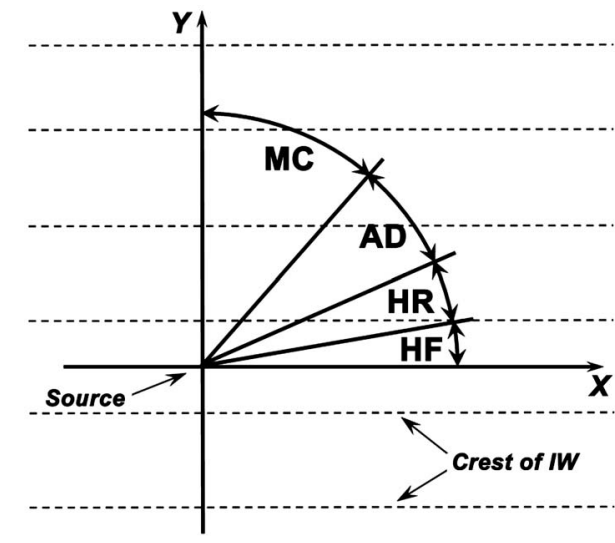

FIG. 1. Schematic diagram showing the regions of acoustic phenomenon occurring in relation to the propagation track relative to the propagation front of the internal waves.

The large sector denoted by MC is the angular region where mode coupling is expected to play a dominant role as a mechanism of sound fluctuations. In this region, the sound signals cross numerous different crests of the ISW train. A simple estimate of the amount of mode coupling one sees in this sector is determined by ratio of the mode cycle distance $L$ (which is roughly the ray cycle distance, plus a small correction for beam displacement) to the quasi-period $\Lambda$ of the ISW, taking into account the changing projection of this length with horizontal angle. We note that if $L \sin \chi / \Lambda \ll 1$, there is no appreciable mode coupling, whereas if $L \sin \gamma / \Lambda \geq 1$, we will have substantial coupling. For typical conditions, $L \leq 1 \mathrm{~km}$ and $\Lambda \sim 600-700 \mathrm{~m}$, so for angles $\chi$ $<45^{\circ}$ we can suppose that mode coupling is small.

The large sector denoted by $\mathrm{AD}$ refers to the region where the propagation is mainly adiabatic. In this region, little mode coupling or horizontal refraction takes place.

The third sector we identify is where the effects of horizontal refraction are significant. This sector is divided into two subsectors. They are referred to as the HF (horizontal focusing) region, where the focusing of the horizontal (modal) rays can occur, ${ }^{10}$ and the HR (horizontal refraction) sector outside of this region, where horizontal refraction still occurs (and is comparatively small) but focusing does not.

It will be shown that in the aforementioned sectors, sound fluctuations have different behavior. For example, in sector $\mathrm{AD}$, the intensity fluctuations, which are caused by the vertical broadening and narrowing of the effective channel by perturbations of the thermocline level, are of the order of 1.5-2 dB. Value of fluctuations in sectors HR and HF are substantially different, as will be discussed later.

In order to clarify the above in terms of horizontal rayvertical mode theory, we next examine the behavior of rays in the HF and HR sectors in Fig. 2. The $X Y$ plane is shown again with the internal wave crests propagating in the $Y$ direction. Two regions are identified. A focusing ray is shown in the HF region. In the HR region, three ray rubes are shown having variable ray tube cross sections $\delta A$. For a homogeneous environment, the rays are the same at all the angles (as shown by the solid line, R1). If we consider a ray tube with cross section $\delta A$ when an acoustic source is at the peak of an internal wave (corresponding to the maximum displacement
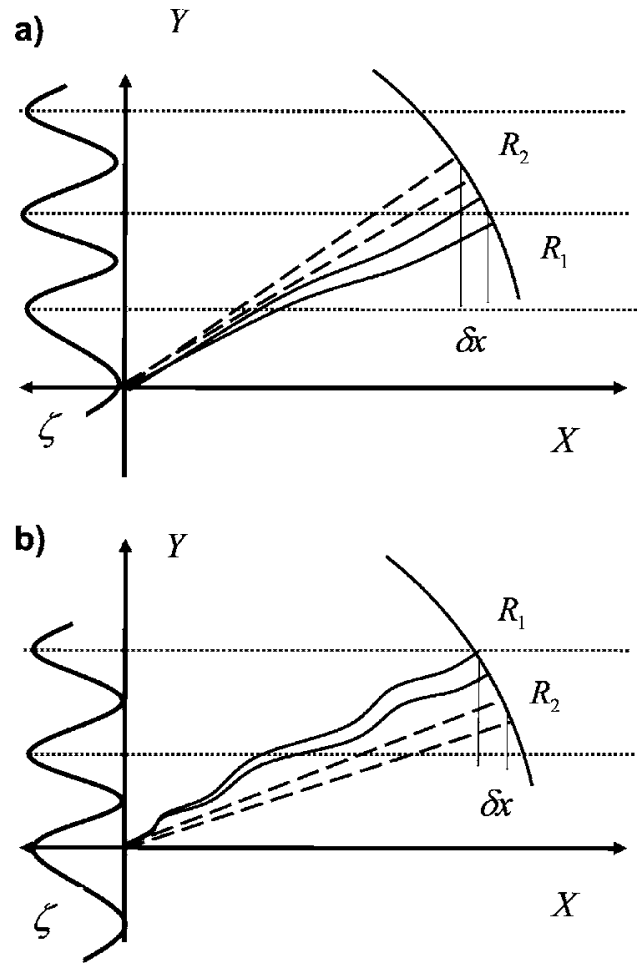

FIG. 2. Schematic diagram showing the behavior of the horizontal ray tubes for sectors HR for different positions of the IW. Positions of ISW, as well as horizontal rays corresponding to "focusing" and "defocusing" situations, are shown by solid lines in (a) and (b) panels. Dotted lines denote crests of internal waves; dashed lines denote horizontal rays in the absence of IW.

of the water layer toward the bottom), we have defocusing, whereas when an acoustic source is in a trough, we have focusing. These two situations can be described in terms of horizontal rays and their ratio can be estimated. The difference between focusing and defocusing is clearly seen in the fluctuations in the data. This was also repeated in our earlier paper. $^{10,11}$

In this paper, a more detailed examination of the ray approximation for horizontal refraction will be presented. In addition, we will perform this analysis with an emphasis on frequency dependence. In our previous paper, we showed fluctuations of the acoustic intensity for a source having a frequency bandwidth of 30 to $160 \mathrm{~Hz}$. Here we will show a wider frequency range, using additional data from an LFM signal that ranged from 50 to $200 \mathrm{~Hz}$.

This paper is organized as follows. In Sec. II the theory of frequency dependent intensity fluctuations due to shallow water internal waves is presented. Then a model is presented for a shallow water channel containing internal waves with characteristics that generally correspond to the SWARM experiment wave field. ${ }^{1-3}$ Next, the experimental data from SWARM'95 are described in detail (Sec. III) for the period of time where the frequency dependence is observed. This is followed by the analysis of these data. Finally, a summary and conclusion section is provided with recommendations for future work.

\section{THEORY OF FLUCTUATIONS AND FREQUENCY DEPENDENCE}

In establishing the theoretical model of sound propagation in shallow water that is used here, we follow the theory 
developed by Ref. 10. We first consider the sound radiated by a point source in the presence of internal solitons in the horizontal plane, using the Weinberg-Burridge horizontal rays/vertical modes approach. We define that the wave fronts of the ISW train are directed along the $X$ axis (Fig. 2). The ISWs are best described by their surfaces of constant density as a function of both spatial coordinates and time. In one popular approximation, the density surface value can be represented as a product of the first gravitational mode of the ISW and an envelope function:

$$
\zeta(\boldsymbol{r}, z, T)=\Phi(z) \zeta_{s}(\boldsymbol{r}, T),
$$

where $\Phi(z)$ is the first gravitational mode, normalized by $\max [\Phi(z)]=1$, and $\zeta_{s}(\boldsymbol{r}, T)$ is the envelope of the internal waves (the displacement of the isodensity surface at the depth where $\Phi$ has a maximum). We will denote the time describing the motion of the solitons as "slow time" and use a capital $T$ for it, in contrast to the "fast time" describing the sound pulse arrival, denoted by $t$. Horizontal position is denoted by $\boldsymbol{r}=(x, y)$. The solitons provide a sound speed profile perturbation free given by

$$
\delta c=Q c_{0}(z) N^{2}(z) \zeta_{s}(\boldsymbol{r}, z, T),
$$

where $c_{0}(z)$ is the unperturbed sound speed profile, $N(z)$ is the buoyancy frequency, and the coefficient $Q$ is a temperature dependent quantity having the value $2.4 \mathrm{~m} / \mathrm{s}$ at $10{ }^{\circ} \mathrm{C}$ and 1.1 for $21^{\circ} \mathrm{C}$.

Our analysis will assume the sound field to be due to a broadband source with spectrum $S(\omega)$, placed at the point $\left(\boldsymbol{r}_{s}, z_{s}\right)$ in the shallow water waveguide. Using the acoustic vertical modes, one obtains for the pressure field

$$
P(\boldsymbol{r}, z, t)=2 \int_{0}^{\infty} S(\omega) \sum_{l} P_{l}\left(\boldsymbol{r}, \boldsymbol{r}_{S}\right) \psi_{l}(\boldsymbol{r}, z) e^{-i \omega t} d \omega,
$$

where the $\psi_{l}$ are the eigenfunctions (modes) and $q_{l}$ and $\gamma_{l} / 2$ are the real and imaginary parts of the eigenvalues $\xi_{l}=q_{l}$ $+i\left(\gamma_{l} / 2\right)$ obtained by solving the Sturm-Liouville problem

$$
\frac{d^{2} \psi_{l}(\boldsymbol{r}, z, T)}{d z^{2}}+\left\{\frac{\omega^{2}}{\left[c_{0}(z)+\delta c(\boldsymbol{r}, z, T)\right]^{2}}-\xi_{l}^{2}(\boldsymbol{r}, T)\right\} \psi_{l}(\boldsymbol{r}, z, T)=0
$$

subject to the usual boundary conditions

$$
\begin{aligned}
& \left.\psi_{l}\right|_{z=0}=0,\left.\quad \psi_{l}\right|_{z=H^{-}}=\left.\psi_{l}\right|_{z=H^{+}}, \\
& \left.\frac{1}{\rho} \frac{d \psi_{l}}{d z}\right|_{z=H^{-}}=\left.\frac{1}{\rho_{1}} \frac{d \psi_{l}}{d z}\right|_{z=H^{+}} .
\end{aligned}
$$

In the above, $\boldsymbol{r}_{s}=(0,0)$ gives the coordinates of the source, $\rho, \rho_{1}$ are the densities of the water and bottom, respectively, and the $P_{l}$ are the mode amplitude coefficients, which are critical to determining horizontal refraction or mode coupling (or both of these phenomena).

Let us examine the $P_{l}$ more closely, neglecting attenuation for the moment. The $P_{l}$ can be found via various different approximations. For instance, they are determined by the horizontal rays in the theory of vertical modes and horizontal rays, or in another variant satisfy the PE equation in the

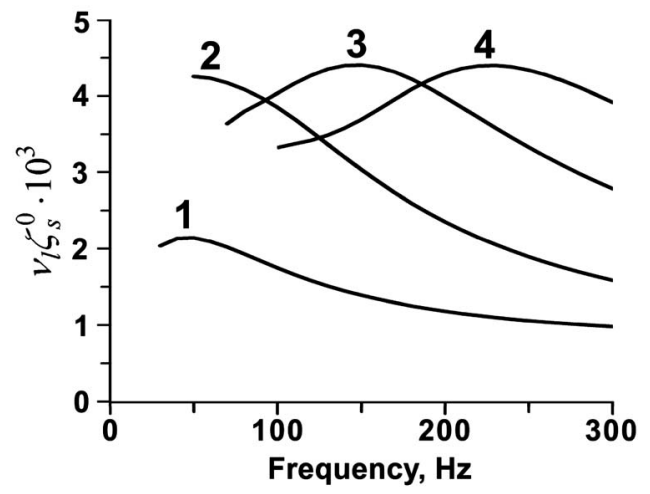

FIG. 3. Frequency dependent index of refraction for horizontal rays at maximum ISW amplitude $(\sim 10 \mathrm{~m})$ calculated for the first four lowest acoustic modes.

theory of vertical modes and PE in the horizontal plane. In a regular waveguide, at a distance far from the source (far field) $\quad P_{l}\left(\bar{r}, \bar{r}_{s}\right)=i \Psi_{l},\left(\boldsymbol{r}_{s}, z_{s}\right) \exp \left(i \xi_{l}^{0}\left|\bar{r}-\boldsymbol{r}_{s}\right|\right) / \sqrt{8 \pi \xi_{l}^{0}\left|\bar{r}-\boldsymbol{r}_{s}\right|}$, where superscript 0 denotes values concerned with unperturbed waveguide (without ISW). In the theory of vertical modes and horizontal rays, we take $P_{l}\left(\bar{r}, \bar{r}_{s}\right)$ to be of the useful form

$$
P_{l}\left(\bar{r}, \bar{r}_{s}\right)=A_{l}(\boldsymbol{r}) e^{i \theta_{l}(\boldsymbol{r})} .
$$

[If we have several horizontal rays corresponding to one mode coming to one receiver point, we should write a second subindex to denote a sum over horizontal rays in (5), see Ref. 10.]

For $A_{l}(\boldsymbol{r})$ and $\theta_{1}(\boldsymbol{r})$, we get the standard eikonal and transport equations of ray theory:

$$
\begin{aligned}
& \left(\nabla_{r} \theta_{l}\right)^{2}=\left(q_{l}^{0}\right)^{2}\left[1+\mu_{l}(\boldsymbol{r})\right], \\
& 2 \nabla_{r} A_{l} \nabla_{r} \theta_{l}+A_{l} \nabla_{r}^{2} \theta_{l}=0 .
\end{aligned}
$$

For the correction to the effective refraction index, $\mu_{1}$, we can employ an expression from perturbation theory, used in Ref. 10.

$$
\begin{aligned}
\mu_{l} & =-\nu_{l} \zeta_{s}(\boldsymbol{r}, T), \\
\nu_{l} & =\frac{2 Q k^{2}}{\left(q_{l}^{0}\right)^{2}} \int_{0}^{H}\left[\psi_{l}^{0}(z)\right]^{2} N^{2}(z) \Phi(z) d z .
\end{aligned}
$$

In our statement of the problem, the index of refraction for horizontal rays depends only on $y$. This $y$ dependence repeats in a regular fashion, as we can see from Eq. (7), via its dependence on the envelope of the ISW train. It is also seen that the coefficient $\nu_{l}$ (and in turn the refractive index for the horizontal rays) corresponding to the $l$ th mode depends on frequency. The details of this frequency dependence were analyzed in Ref. 10, and, as an example, we show the frequency dependence of the horizontal refraction index (HRI) for some modes and for conditions corresponding to the SWARM'95 experiment (see Fig. 3). As a result of the index of refraction frequency dependence, we will also observe frequency dependence of the sound intensity, and in particular a frequency dependence of sound intensity fluctuations. For the SWARM'95 experiment, this frequency dependence 
of the intensity fluctuations was discussed in Ref. 6, where it was remarked that the shape of this dependence repeats the shape of the frequency dependence of the refraction index. Below we explain this interesting fact in more detail.

Let us consider intensity fluctuations using the familiar approximation of horizontal rays/vertical modes. This model corresponds to the one presented in Ref. 6. We assume that the internal solitons have plane wavefronts, parallel to the $X$ axis, and moving in the $Y$ direction at the velocity $V$, i.e., $\zeta_{s}(\boldsymbol{r}, T)=\zeta_{s}\left(\boldsymbol{r}_{R}, T+\left(y-y_{R}\right) / V\right)$. Here $\boldsymbol{r}_{R}=\left(x_{R}, y_{R}\right)$ is the coordinate of the receiver in the horizontal plane. We will also assume (not strictly correctly) that the shape of the ISW envelope does not change in time, and will denote it as $\zeta_{s}(y, T)$. In our approach, the parameter $\mu_{l}$ determining the medium of propagation is a function only of $y: \mu_{l}=\mu_{l}(y)$. This greatly simplifies our consideration of the ray pattern.

In order to carry out calculations, we need to construct expressions for the horizontal rays. In our formalism, for every mode number $l$ we have a system of horizontal rays (i.e., their trajectories). Thus rays belonging to this system will be characterized both by mode number and by their horizontal launch angle. It is useful to define the tangent vector to this trajectory; in particular, at the source this vector is $\tau_{l s}=\left(\cos \chi_{l s}, \sin \chi_{l s}\right)$, where $\chi_{l s}$ is the angle of the horizontal ray with the $x$ axis, corresponding to the $l$ th vertical mode at the point of the source. The trajectories of the horizontal rays (HR), which are outgoing from the source [point $(0,0)]$, can be described by an equation at the current point at the ray $(x, y)$ as $y=y(x)$ or $x=x(y)$. We can also use other parameters and characterize rays by their so-called ray coordinates - the launch angle and the length of the raypath: $\left(\chi_{l s}, s\right)$, where the parameter $d s=\sqrt{d x^{2}+d y^{2}}$ is an element of arc along the ray.

We are interested here mainly in the fluctuations of intensity of the sound field. For a broadband signal, we can introduce the spectral-modal intensity

$$
\boldsymbol{I}_{l \omega}=\frac{2 \pi i}{\rho \omega}|S(\omega)|^{2}\left(P_{l} \nabla P_{l}^{*}-P_{l}^{*} \nabla P_{l}\right) .
$$

In the approximation of a locally plane wave front $\left|\nabla P_{l}\right|$ $\sim k P_{l}$, we obtain a simpler expression

$$
\boldsymbol{I}_{l \omega}=\frac{4 \pi}{\rho c}|S(\omega)|^{2}\left|P_{l}\right|^{2}
$$

In ray theory, the amplitude $\left|P_{l}\right|=A_{l}$ of the separate HRs can be calculated as

$$
A_{l}(s)=\frac{A_{l}\left(s_{0}\right)}{\sqrt{D_{l}\left(s, s_{0}\right)}},
$$

where $A_{l}\left(s_{0}\right)$ is amplitude of ray at a fixed point (usually taken as near the source) and $D_{l}\left(s, s_{0}\right)$ is the divergence of the horizontal ray tube. This can be presented more elegantly through the Jacobian $J(s)=\partial(x, y) / \partial\left(s, \chi_{l s}\right)$ of the transformation from Cartesian coordinates to ray coordinates $x=x\left(s, \chi_{l s}\right), y=y\left(s, \chi_{l s}\right)$ :

$$
D_{l}\left(s, s_{0}\right)=\frac{J(s)}{J\left(s_{0}\right)}
$$

Thus the spectral modal intensity, propagating along horizontal ray, as a function of distance is

$$
I_{l \omega}=\frac{4 \pi}{\rho c}|S(\omega)|^{2}\left(A_{l}^{0}\right)^{2} \frac{J\left(s_{0}\right)}{J(s)}=I_{l \omega}^{0} \frac{J\left(s_{0}\right)}{J(s)},
$$

where $I_{l \omega}^{0}$ is some reference value of the modal intensity. Its value and the value of the Jacobian at the reference distance $J\left(s_{0}\right)$ do not play an essential role because, in the following considerations, we will focus on relative fluctuations of the intensity.

Due to the changing position of the source relative to the ISW train, we observe different intensity behaviors of the horizontal rays as the ISW wave train passes by. Using Fig. 2, we will next examine intensities at two limiting positions of the internal solitons, corresponding to strong focusing and defocusing of the horizontal rays. Due to the motion of the internal solitons, we observe variation of the ray pattern between these two structures. Let us first consider an area HF where the focusing of horizontal rays can take place, and thus fluctuations of the ray trajectories are significant. For this case, ray trajectories can be constructed numerically for any arbitrary shape of ISW. We can estimate the boundary of this area if we know the parameters of the ISW train. Let the ISWs have some amplitude $\zeta_{0}$, typically $\sim 10 \mathrm{~m}$, and quasiperiod $\Lambda$, typically $\sim 700 \mathrm{~m}$. This simple estimate gives a limiting horizontal angle for outgoing rays $\chi_{0} \sim \sqrt{\left|\nu_{l} \zeta_{0}\right|} \sim 4^{\circ}$. Sound fluctuations in this area $(\mathrm{HF})$ can be significant and depend on the mode number, frequency, and position of the receiver-thus they cannot be simply estimated. Numerical calculations for distances $\sim 15 \mathrm{~km}$ (similar to the SWARM'95 experiment) give amplitude fluctuations of $\geq 7-10 \mathrm{~dB}$. Concerning defocusing, we would remark that if position of the source corresponds to this case, then the horizontal rays go away from the area between two adjacent solitons and so in this area there is a shadow zone.

Let us next consider the HR region $\left(\chi>\chi_{0}\right.$, with an upper boundary that we will determine later). Ray trajectories (denoted by dashed lines in Fig. 2) in this area approximately follow the straight lines, which would be seen in the absence of internal waves. We can thus construct the actual trajectory by using this small deviation to justify a perturbation approach.

Of prime importance to us here is the estimation of intensity fluctuations due to horizontal refraction. We will do this estimation using the ray theory transport equation [Eq. (13)] and calculating the divergence of the horizontal rays or, in other words, simply calculating the cross section of the two-dimensional ray tube. Let us now consider rays outgoing from the source, which, as we said, are determined by the connection between the $x$ and $y$ coordinates at the current point of the ray $y=y(x)$ or $x=x(y)$. We will omit the index $l$, assuming for now that a given HR is for a given vertical mode $l$. According to Snell's law 

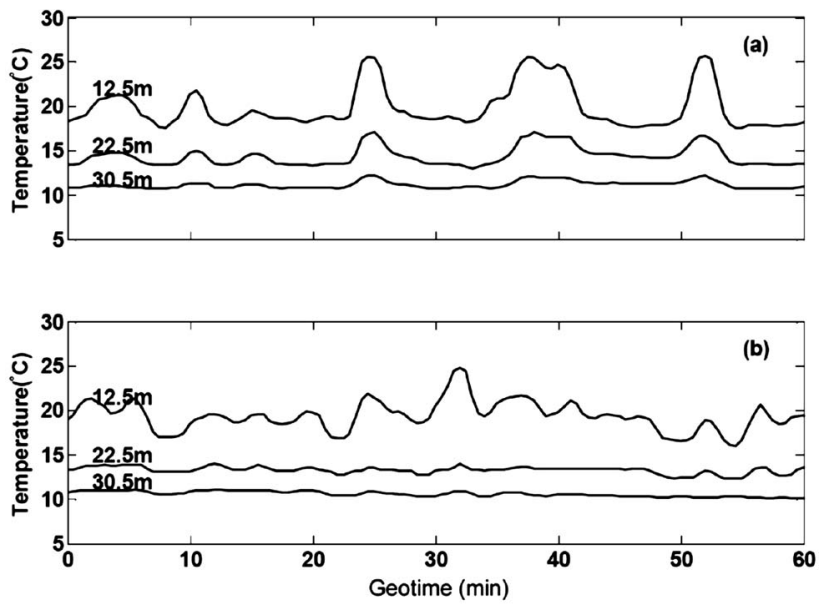

FIG. 4. Recorded temperature data at WHOI vertical array on 4 August 1995. (a) From 19:00:00 to 20:00:00 GMT (case 1). (b) From 20:00:00 to 21:00:00 GMT (case 2). Depths of the corresponding sensors are denoted near the curves.

$$
\frac{\cos \chi_{l s}}{\cos \chi_{l}(y)}=1+\mu_{l}(y) / 2
$$

and

$$
\frac{d x}{d y}=\cot \chi_{l}(y)
$$

so we have

$$
x=\cos \chi_{l s} \int_{0}^{y} \frac{1}{\sqrt{\mu_{l}(y)+\sin ^{2} \chi_{l s}}} d y .
$$

This should work, since for internal solitons, $\left|\mu_{l}\right| \approx 10^{-3}$. Thus our approximation is true for $\chi_{l s} \geq 2-3^{\circ}$; for values less than this, we would need to use another approach. For small perturbations, but not small angles, $\left|\mu_{l}(y)\right| \ll \sin ^{2} \chi_{l s}$, so we have

$$
x=y \cot \chi_{l s}-\frac{\cos \chi_{l s}}{2 \sin ^{3} \chi_{l s}} \int_{0}^{y} \mu_{l}(y, T) d y .
$$

Using Eq. (7) we have

$$
x=y \cot \chi_{l s}+\frac{\nu_{l}(\omega) \cos \chi_{l s}}{2 \sin ^{3} \chi_{l s}} \int_{0}^{y} \zeta_{s}(y, T) d y .
$$

The second term on the right-hand side of Eq. (18) is the correction to the straight line path, which is the first term, corresponding to absence of IW [dashed lines in Figs. 2(a) and 2(b)]. Remark that in dependence on $y$ coordinate of the source $(y=0)$ with respect to the envelope, the integral on the right side of (18) can have a different sign. More exactly, if point $y=0$ corresponds to the minimum of the soliton, then the integral in (18) has positive sign and we have the $\delta x$ $>0$ "focusing" situation [Fig. 2(a)]. If point $y=0$ falls to the maximum of the soliton, the integral has negative sign - the defocusing situation [Fig. 2(b)].

Let us now carry out the calculations of the ray trajectory and the ray intensity (cross section of the ray tube) as influenced by horizontal refraction. Let us consider rays emitted from the source at angles greater than the critical
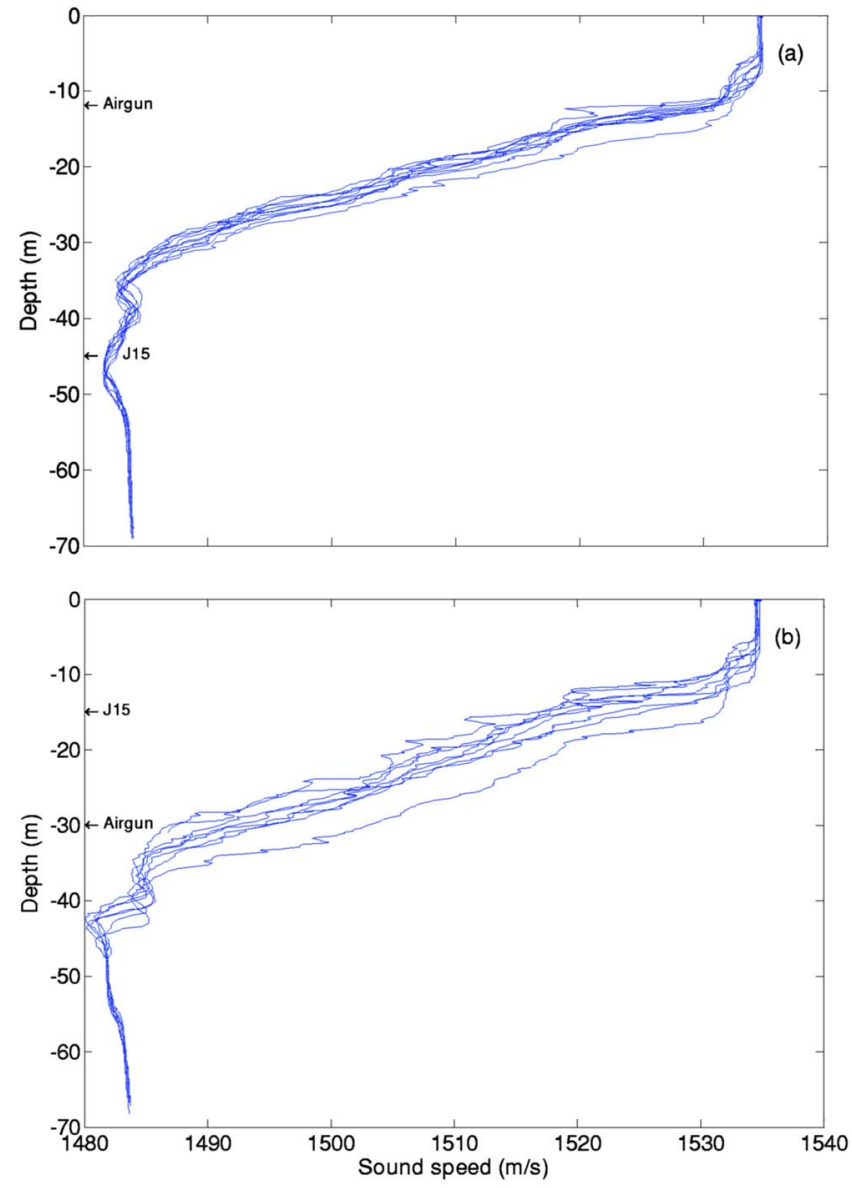

FIG. 5. (Color online) Sound speed profiles, corresponding to different geotimes at the source position, (a) case 1, (b) case 2. Depths of the sources are denoted by arrows.

angle for horizontal focusing $\chi_{l s}>\chi_{0}$. For this case, the approximate ray path is shown in Fig. 2 . The horizontal deviation of the ray is denoted by $\delta x$. We can see that this deviation is determined by the integral of the envelope of the solitons, simply speaking the area under the curve. Let us assume the simplest shape for the solitons (a box), so that this area can be estimated as

$$
\int_{0}^{y} \zeta(y, T) d y \sim \frac{y \zeta_{0}}{2}
$$

(here we suppose that "focusing" takes place) and so

$$
x=y \cot \chi_{l s}+\frac{\nu_{l}(\omega) \zeta_{0} \cos \chi_{l s}}{4 \sin ^{3} \chi_{l s}} y=y \cot \chi_{l s}\left(1+\frac{\nu_{l}(\omega) \zeta_{0}}{4 \sin ^{2} \chi_{l s}}\right) .
$$

Thus the ray that would go to the point $R_{1}$ in the unperturbed case arrives at $R_{2}$. Without internal waves, the signal propagates along the corresponding radius vector.

Now let the unperturbed length be determined by the parameter $s=\sqrt{y^{2}+(x+\delta x)^{2}}$. The intensity at the receiver point without internal waves is determined only by the distance to the source and does not depend on the launch angle in the horizontal plane. The equation for the parameter $s$ can be written in the form 

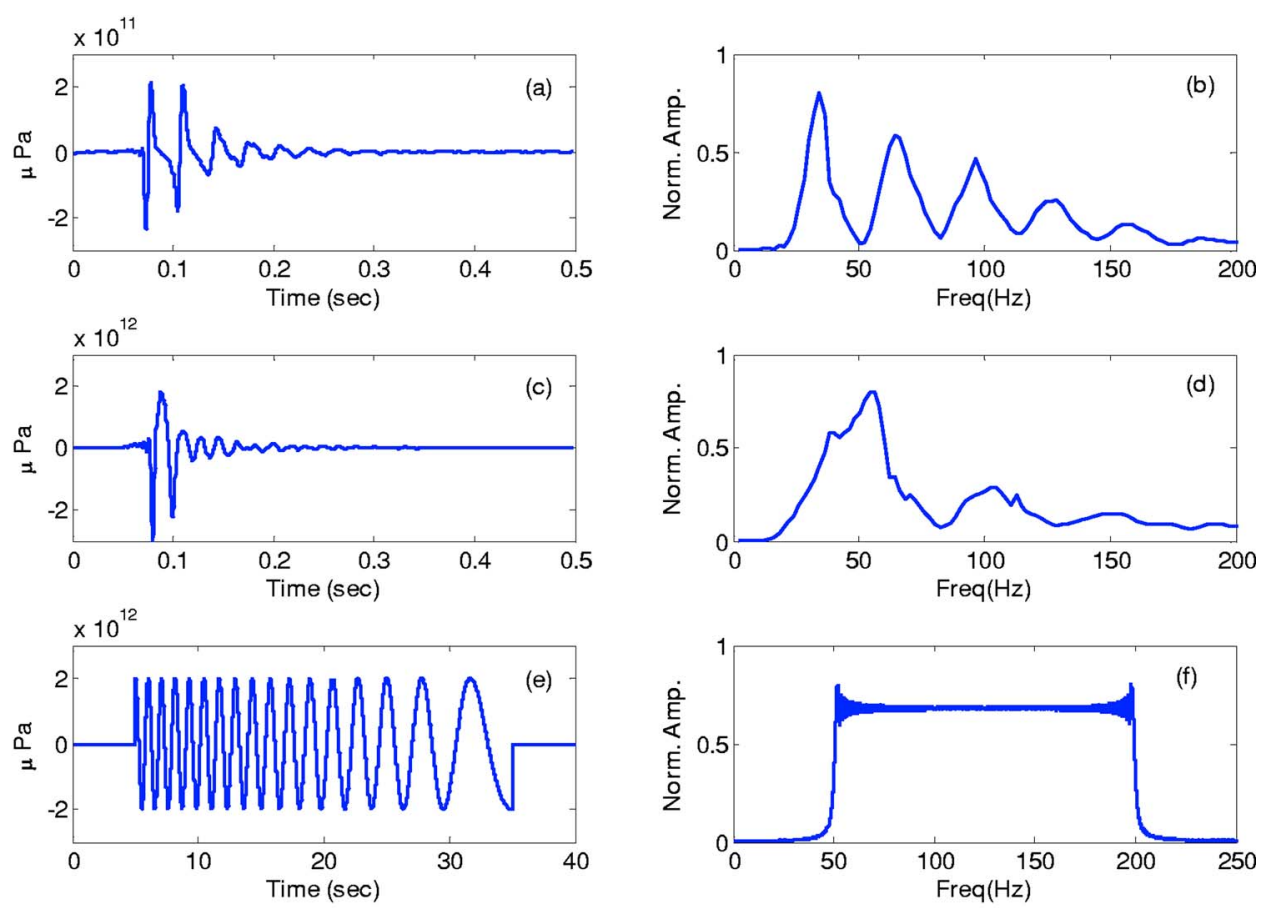

FIG. 6. (Color online) Recorded sound sources signatures measured at $1 \mathrm{~m}$. (a) Airgun at $12 \mathrm{~m}$ depth from the sea surface. (b) Airgun spectrum at $12 \mathrm{~m}$ depth. (c) Airgun at $30 \mathrm{~m}$ depth. (d) Airgun spectrum at $30 \mathrm{~m}$ depth. (e) J15 at $15 \mathrm{~m}$ depth transmitting LFM sweep. (f) LFM signal spectrum at $15 \mathrm{~m}$ depth. Note that while the airgun signature is depth dependent due to the bubble pulse, the J15 signal is independent of the depth.

$$
\begin{aligned}
s(y) & =\int_{0}^{y} \frac{d s}{d y} d y=\int_{0}^{y} \sqrt{\cot ^{2} \chi(y)+1} d y \\
& =\int_{0}^{y} \frac{1+\mu_{l}(y)}{\sqrt{\mu_{l}(y)+\sin ^{2} \chi_{s}}} d y .
\end{aligned}
$$

Using the smallness of the perturbation to the refraction index

$$
\begin{aligned}
s \approx & \frac{1}{\sin \chi_{l s}} \int_{0}^{y}\left(1+\frac{\mu_{l}}{2 \sin ^{2} \chi_{l s}}\right) d y=\frac{1}{\sin \chi_{l s}} y \\
& +\frac{\nu_{l} \zeta_{0}}{4 \sin ^{3} \chi_{l s}} y,
\end{aligned}
$$

and so we get

(a)
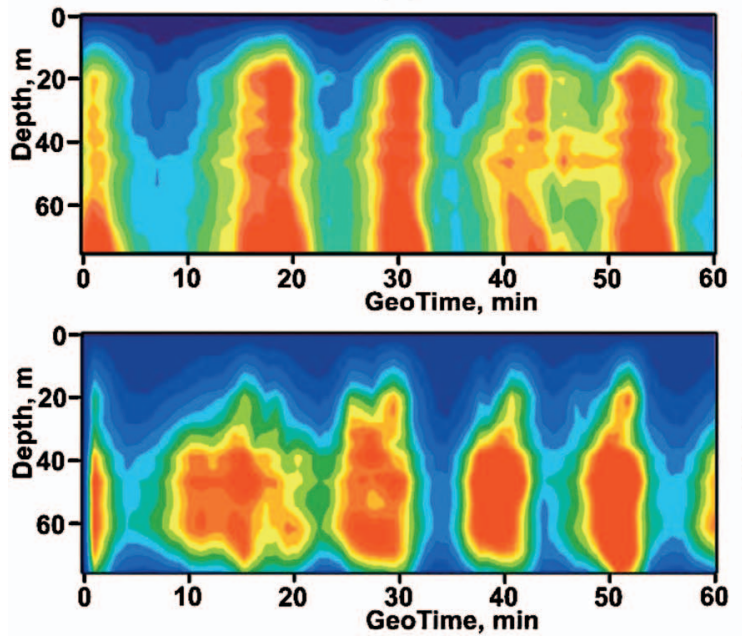

$$
y \approx s\left(\sin \chi_{l s}-\frac{\nu_{l} \zeta_{0}}{4 \sin \chi_{l s}}\right) .
$$

We can see connection between $x$ and $s$. In the same approximation

$$
x \approx s \cos \chi_{s}
$$

and the Jacobian at the point $R_{2}$ can be expressed as

$$
J(s)=\frac{\partial(x, y)}{\partial\left(s, \chi_{l s}\right)} \approx s\left[1+\frac{\nu_{l} \zeta_{0} \cos 2 \chi_{l s}}{4 \sin ^{2} \chi_{l s}}\right] .
$$

We can now get the intensity in accord with Eq. (13) using the assumption of small grazing angles at the point $R_{2}$. For the case of focusing, it is decreasing (in correspondence with experimental data) and at a fixed $s$

(b)
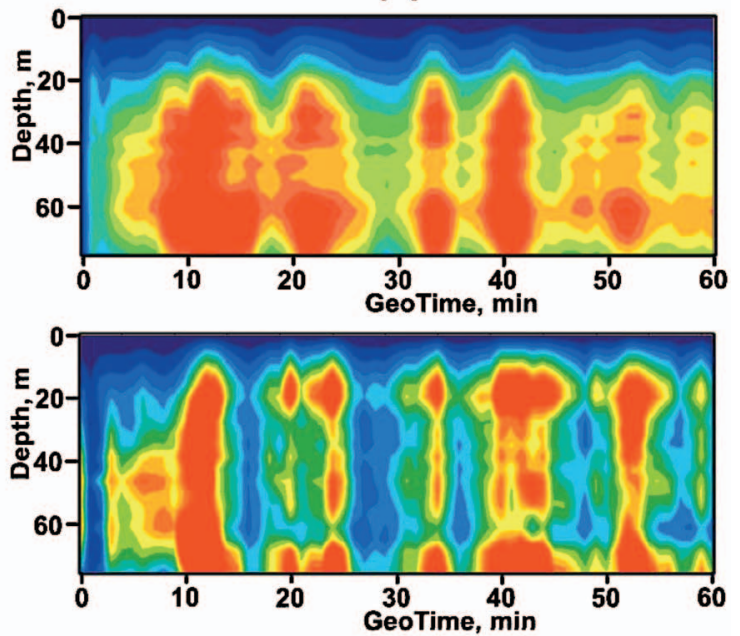

FIG. 7. Fluctuations of sound energy per pulse in normalized units for airgun (top) and J15-LFM (bottom) sources. (a) T=19:00-20:00 GMT, (b) $T=20: 00-21: 00$ GMT. Positions of the sources for the cases (a) and (b) are shown in Fig. 5. 

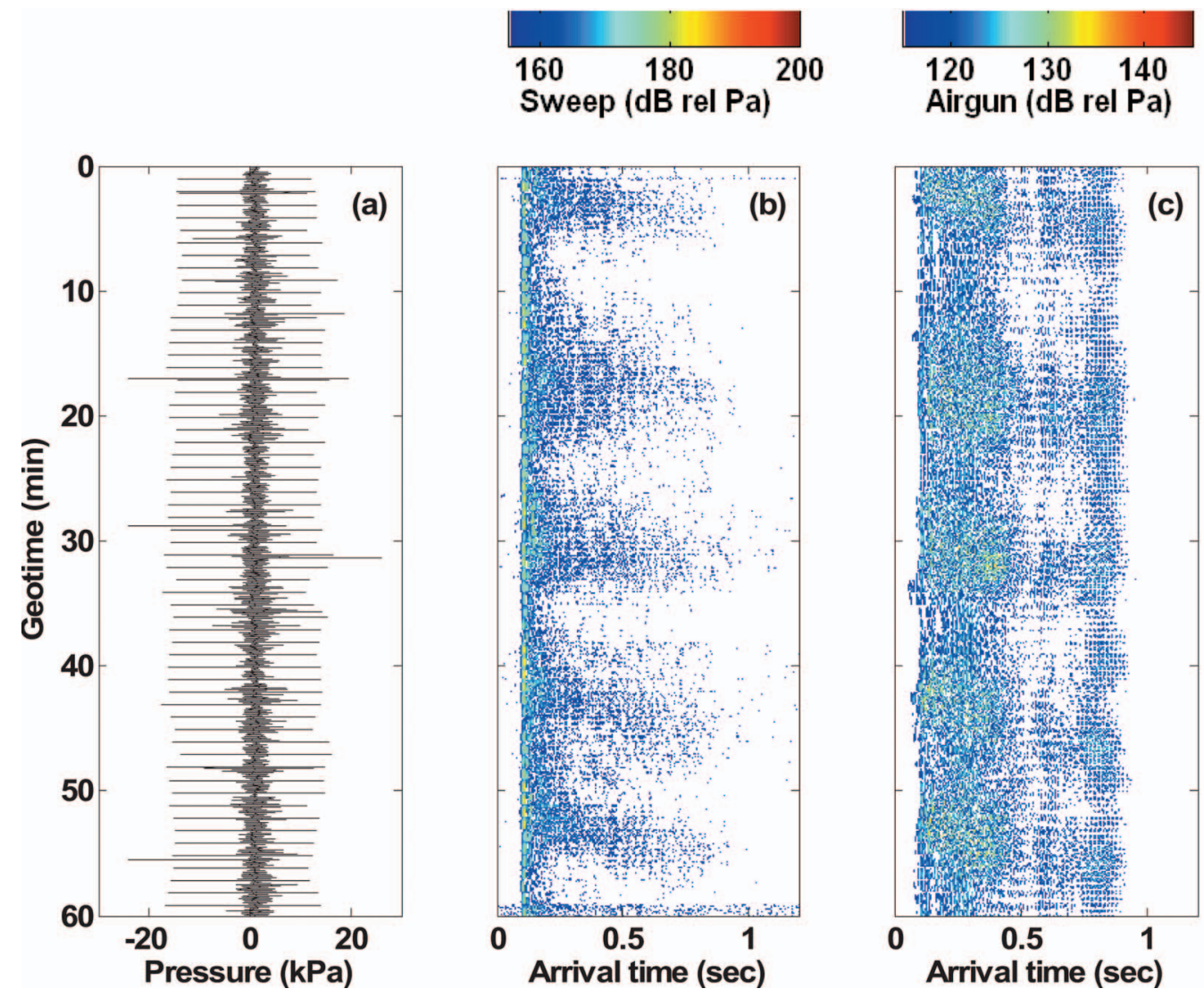

FIG. 8. Acoustic intensity versus geotime, $T$ measured by the WHOI VLA for case 1. (a) Airgun and LFM signatures, (b) intensity of the received airgun source when placed $12 \mathrm{~m}$ below the sea surface (above thermocline), and (c) LFM source when placed below thermocline at $45 \mathrm{~m}$ below the sea surface.

$$
I_{l \omega}^{f}=I_{l \omega}^{0} J\left(s_{0}\right)\left(1+\frac{\nu_{l}(\omega) \zeta_{0}}{4 \sin ^{2} \chi_{l s}}\right)^{-1}
$$

For defocusing, the divergence of rays is decreasing, and so the intensity is correspondingly increasing and proportional to the value

$$
I_{l \omega}^{d}=I_{l \omega}^{0} J\left(s_{0}\right)\left(1-\frac{\nu_{l}(\omega) \zeta_{0}}{4 \sin ^{2} \chi_{l s}}\right)^{-1}
$$

In particular, for solitons with amplitude $\sim 15 \mathrm{~m}$, a value of $\nu_{l} \sim 5-7 \times 10^{-4}$ and grazing angle $\sim 0.1\left(6^{0}\right)$, we have $(5-7$ $\left.\times 10^{-4} \times 15\right) / 4 \times 0.01 \sim 0.2-0.3$ and so the ratio of intensities is $I^{f} / I^{d} \sim 1.8-2$.

Due to the motion of the ISWs, a fixed source and receiver fall into positions first corresponding to the focusing of horizontal rays and then corresponding to defocusingthus we observe temporal fluctuations of the received signals. We next estimate these fluctuations as a function of the parameters of the problem.

As to the frequency dependence of the fluctuations, this can be seen from the expression for the correction $\delta I_{l \omega}^{2}=I_{l \omega}^{2}$ $-\left\langle I_{l} \omega\right\rangle^{2}$. We will consider the value of the scintillation index SI (Ref. 12) describing relative fluctuations of intensity between maximal (defocusing) and minimal (focusing) values to be:

$$
\mathrm{SI}_{l \omega}^{2}=\left\langle\frac{\delta I_{l \omega}^{2}}{\left\langle I_{l \omega}\right\rangle^{2}}\right\rangle=\frac{\left\langle I_{l \omega}\right\rangle^{2}-\left\langle I_{l \omega}\right\rangle^{2}}{\left\langle I_{l \omega}\right\rangle^{2}} .
$$

Here $\langle F\rangle=1 / T \int_{0}^{T} F d T$. We note that in parts of the literature, the SI is sometimes defined without the squared expression. We are following the (squared) convention of Ref. 12 in this work.

Thus, for our case, the scintillation index is

$$
\mathrm{SI}_{l \omega}^{2} \sim \frac{\nu_{l}(\omega) \zeta_{0}}{\sin ^{2} \chi_{s}}
$$

According to this equation, the frequency dependence of the scintillation index for modal intensity repeats the shape of the frequency dependence of the horizontal refraction index for the same mode. This dependence is shown in Fig. 3.

In the following sections, we will analyze experimental results for SI, obtained in the SWARM'95 experiment, and compare to the theory developed.

\section{DATA ANALYSIS AND COMPARISON WITH THEORY}

To test the theory presented in previous sections, we show here an analysis of data obtained in the SWARM-95 experiment, to compare with the theory. Since several papers have previously outlined details of these measurements, ${ }^{1-3}$ we do not describe the minutiae of the experiment. Here we only describe $2 \mathrm{~h}$ of collected data during 4 August 1995 . 

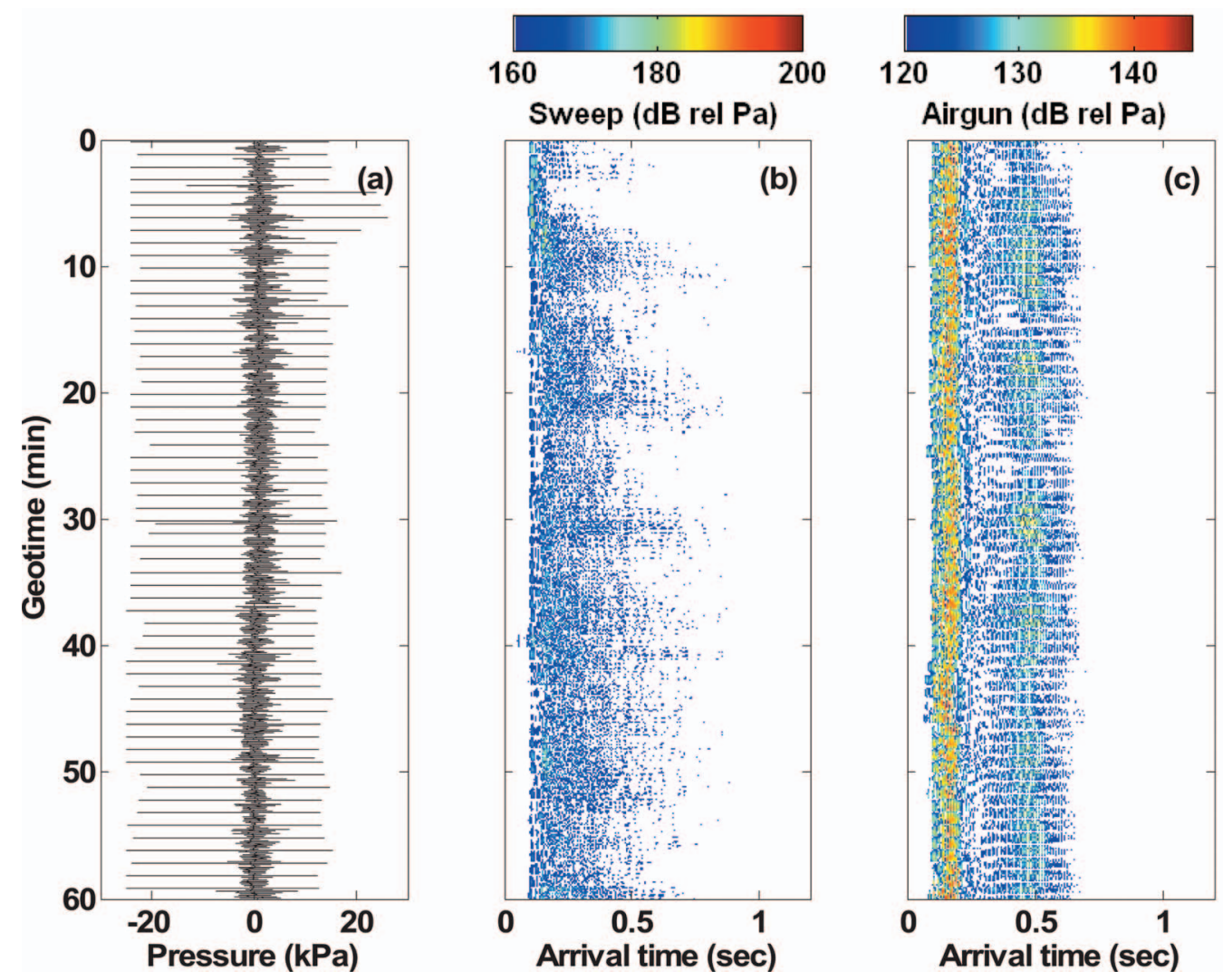

FIG. 9. Acoustic intensity versus geotime, $T$ measured by the WHOI VLA for case 2. (a) Airgun and LFM signatures, (b) intensity of the received airgun source when placed $30 \mathrm{~m}$ below the sea surface (below the thermocline), and (c) LFM source when placed above thermocline at $15 \mathrm{~m}$ below the sea surface.

During each hour, two acoustic sources having different frequency bands (an airgun at 30-150 Hz, and a J15-3 transducer at $50-200 \mathrm{~Hz}$ ) were used to simultaneously produce sound in the waveguide. One of the acoustic sources was placed above the thermocline while the second one was placed below. The airgun source fired every $60.03 \mathrm{~s}$ while the J15 was transmitting an LFM signal of duration $30 \mathrm{~s}$ in between two consecutive airgun shots. The data are presented in two geotime segments-one segment from 19:00 to 20:00 GMT that we call case 1, and the second segment from 20:00 to 21:00 GMT called case 2 .

Figure 4 shows thermistor string records for these two time segments at the receiver array. From the six thermistors that were deployed along the vertical line array of the Woods Hole Oceanographic Institution (WHOI-VLA) only the upper water column shows the ISW activity, hence data for depths of $12.5,22.5$, and $30.5 \mathrm{~m}$ below the sea surface are only shown here. The temperature records show strong variability in the upper water column, with a decreasing trend at lower depths. In addition to these temperature records, the sound speed profile was measured using a CTD at the source location at 15-min intervals during the propagation. Although not shown here, these data were used in order to get approximate values for the salinity profiles, which were rather constant during this period. Figure 5 shows the sound speed profiles at the source for the 2-h time intervals with the source positions designated at different depths. During the first hour, the airgun was placed at $12 \mathrm{~m}$ from the sea surface while the J15 was at 45-m depth. During the second hour, the airgun was at $40 \mathrm{~m}$ depth while the $\mathrm{J} 15$ was placed at $15-\mathrm{m}$ depth. We note that there is some change in the spectrum of the airgun due to the depth change, but not the J15.

In Fig. 6 we present the source signals measured about $1 \mathrm{~m}$ from the source position in both cases. It is noted that the signals are very different from each other. While the airgun signal has a time domain duration of $\sim 0.4 \mathrm{~s}$ and energy up to $150 \mathrm{~Hz}$, most of its low frequency energy components peak around $30 \mathrm{~Hz}$. The J15-3 generated a 30-s LFM signal in time domain and its energy is between 50 and $200 \mathrm{~Hz}$.

In order to address the data analysis based on our theory, we first show the temporal dependence of the intensity integrated over the pulse duration. This dependence shows the fluctuation of signals from the acoustic sources for each geotime segment at the WHOI receiver array. ${ }^{2,6}$ Figure $7(\mathrm{a})$ shows the first geotime segment ( $T=19: 00-20: 00$ GMT) when the airgun was placed at $12 \mathrm{~m}$ below the sea surface, and the J15 was placed at $45 \mathrm{~m}$ [see Fig. 5(a)]. The LFM signals presented are seen after matched filter processing and the airgun signal being bandpassed filtered between 20 and $300 \mathrm{~Hz}$. In Fig. 7(b) the second geotime segment ( $T=20: 00-21: 00$ GMT) is shown, when the J15 source is at $15-\mathrm{m}$ and the airgun is at $30-\mathrm{m}$ depth, respectively. It is noticed that the behavior of the waveguide is very similar regardless of the location of the sound source in the water column and for the frequencies of the different sources. Similar results have been reported earlier to relate the intensity fluctuations of the sound signals to the index of refrac- 
(a)

Phone

\#

1
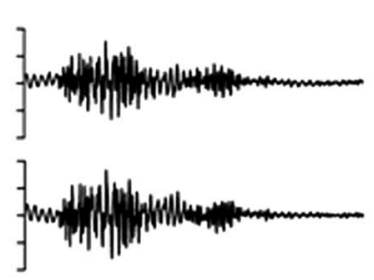

3

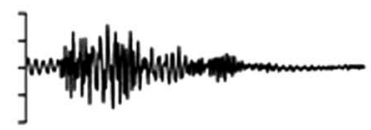

4

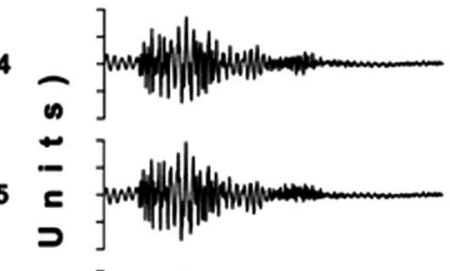

6

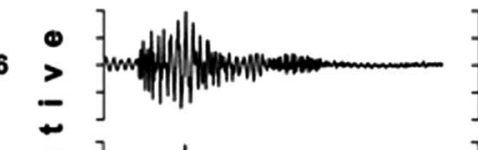

7

7

8

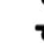

9

10

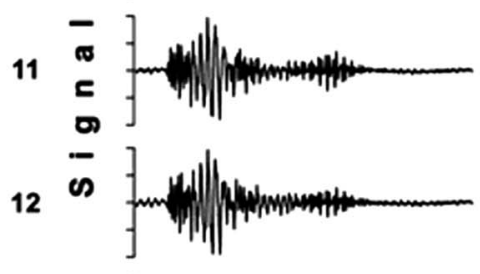

13 ]
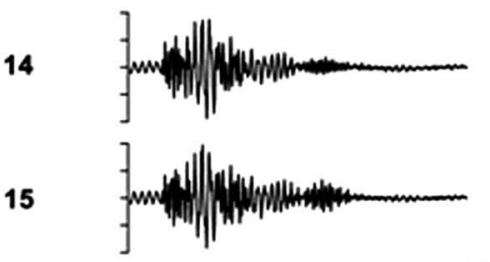

16

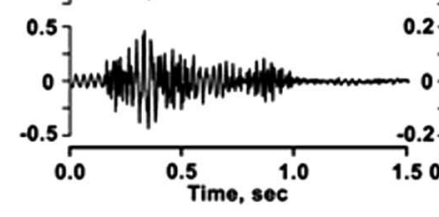

(b)
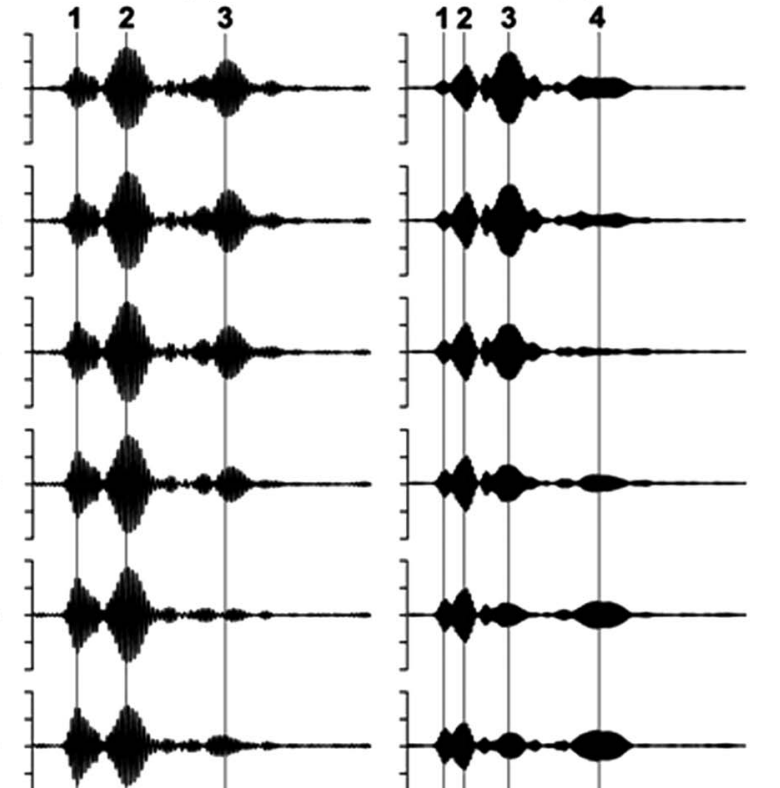
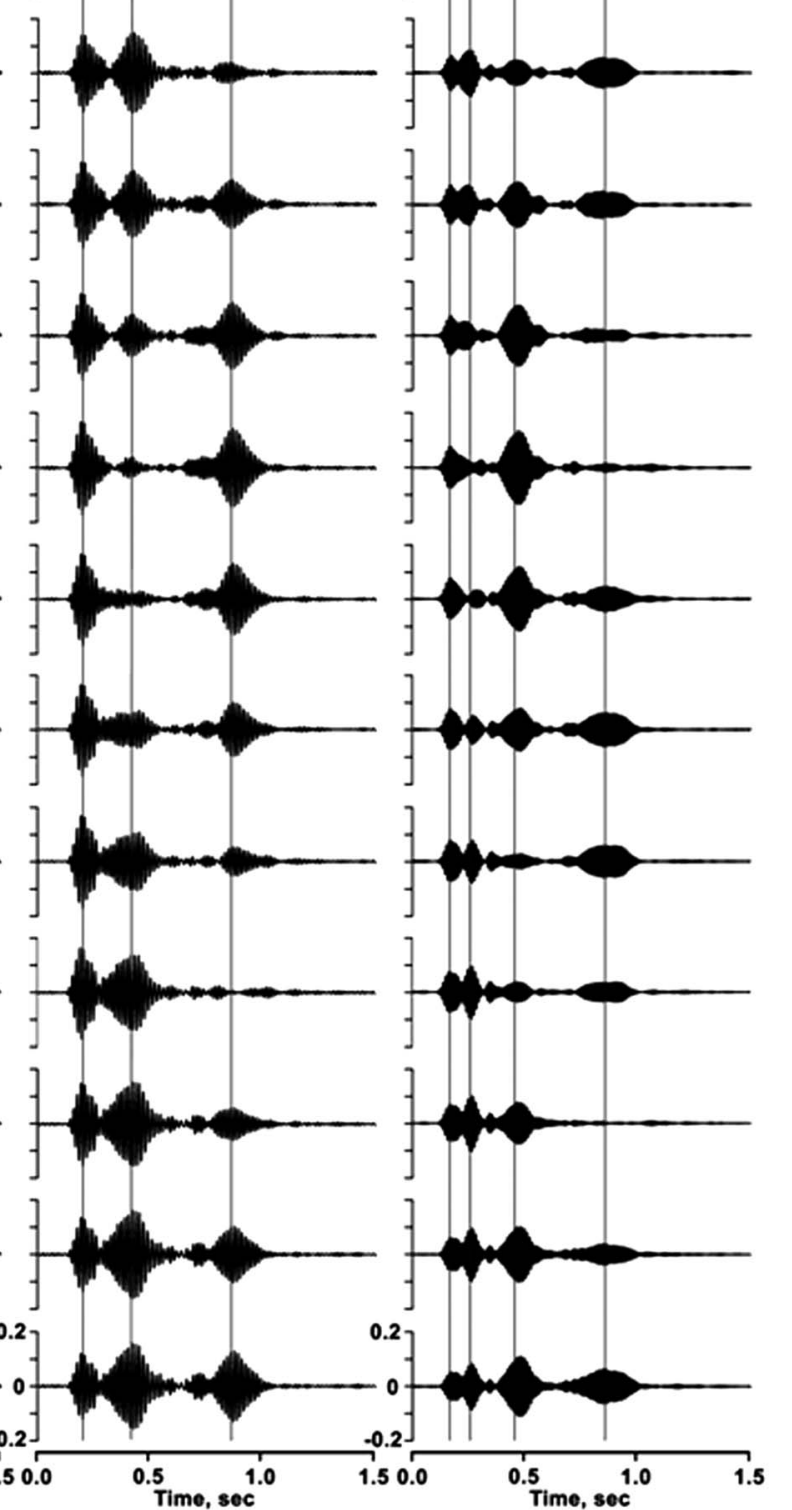

FIG. 10. Received airgun signal for $T=-19: 15$ GMT for all 16 WHOI hydrophones. (a) Raw data. (b) Bandpass filter with fc $=60 \mathrm{~Hz}$ and BW= $\pm 10 \mathrm{~Hz}$. (c) Bandpass filter with $\mathrm{fc}=90 \mathrm{~Hz}$ and $\mathrm{BW}= \pm 10 \mathrm{~Hz}$. Dashed lines indicate arrival times of modes 1-4. 


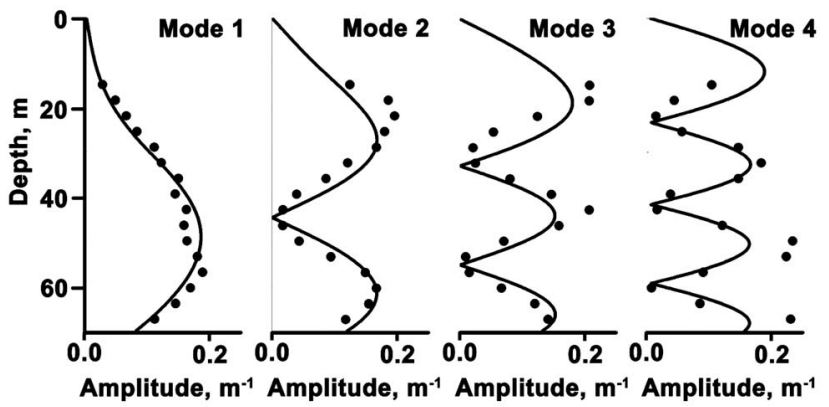

FIG. 11. Depth dependence of the first four mode functions for $T=19: 00-20: 00$ GMT. Dots show averaged value of experimental data and the solid lines show the theoretical calculations based on averaged sound speed profiles for the same time.

tion. Here, we present a more through formalism in the context of experimental verification of the theory.

To further investigate the experimental data, we present the received signals for cases 1 and 2 as a summary of the VLA in the following manner. We compress the received signals for all the elements in the WHOI vertical array into a single point and then stack these points in geotime for each segment of the 2-h period (i.e., cases 1 and 2 shown in Figs. 4 and 5, respectively). As a result, Figs. 8 and 9 are obtained. In each of these figures, three panels are shown. In Fig. 8(a) the source signature measurement near the source is displayed as a function of geotime for case 1. Spikes show the airgun shots and in between; the darker color signals with lower amplitude and longer duration show the LFM sweeps. In Figs. 8(b) and 8(c) the squeezed and stacked array results as a function of geotime are displayed for the source placed above and below the thermocline [Fig. 8(b) shows the received signal at the vertical array for the LFM at $45 \mathrm{~m}$ and Fig. 8(c) shows the received signal at VLA for the airgun at $12 \mathrm{~m}$ ]. Hence, Fig. 8 is a summary of the simultaneous channel response function for the channel activated above and below the thermocline for $1 \mathrm{~h}$ (i.e., case 1 from 19:00 to 20:00 GMT). Similarly, Fig. 9 shows the same results for the second hour of our data with the corresponding source positions shown in Fig. 5 (i.e., case 2 from 20:00 to 21:00 GMT).

Several points are noteworthy in these two figures. The most significant feature of these data is their intensity fluctuations as a function of geotime. We see that the number of oscillations of intensity and the typical temporal period between adjacent fluctuations are approximately the same in Figs. 8 and 9 on one side and in Fig. 4 on the other side. It indicates a direct relationship of the sound fluctuations with the internal waves. The duration of the received signal in the second hour [case 2 shown in Figs. 9(b) and 9(c)] is much shorter compared to the first hour [case 1 shown in Figs. 8(b) and $8(\mathrm{c})$ ]. Also as expected, when the source is placed below the thermocline, the received signal depicts a much stronger intensity [Figs. 8(c) and 9(c)].

Next, the temporal dependence of the intensity contained in separate modes per unit frequency interval [called spectral-modal density $I_{l \omega}$ in Eq. (10)] is extracted from the experimental data and is compared with the scintillation index (29). A pulse signal received by vertical array can be shown as $p(\boldsymbol{r}, z, t)$, where $\boldsymbol{r}$ is horizontal coordinate of the

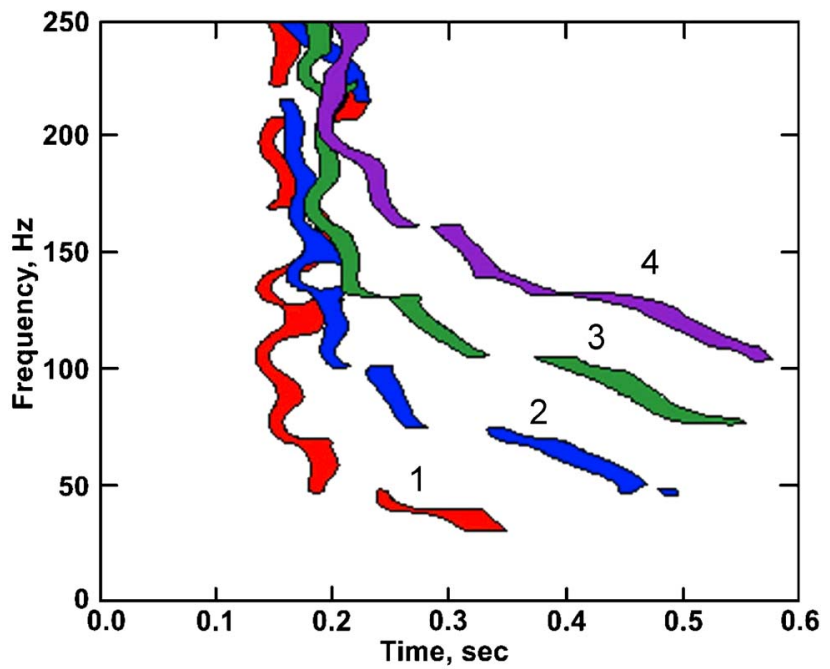

FIG. 12. (Color online) Time-frequency diagram for an airgun pulse at $T$ =19:15 GMT showing the first four modes obtained from the experimental data. Modes 1 through 4 are numbered on the curves.

receiver and $z$ is the hydrophone depth. Since only a discrete number of hydrophones exist, the sound pressure is known only at fixed depths $z_{j}$ and ranges $\boldsymbol{r}_{j}$. However, for simplicity, in the following analysis we omit the index. The spectrum of the received signal $S(\boldsymbol{r}, z, \omega)$ also depends on the hydrophone and analytic (complex) received signal, and has the form

$$
\begin{aligned}
& P(\boldsymbol{r}, z, t)=2 \int_{0}^{\infty} S(\boldsymbol{r}, z, \omega) e^{-i \omega t} d \omega, \\
& S(\boldsymbol{r}, z, \omega)=\frac{1}{2 \pi} \int_{-\infty}^{\infty} \operatorname{Re}[P(\boldsymbol{r}, z, t)] e^{i \omega t} d t .
\end{aligned}
$$

According to our theory, it can be decomposed over vertical modes

$$
S(\boldsymbol{r}, z, \omega)=\sum_{l} S_{l}(\boldsymbol{r}, \omega) \psi_{l}(z)
$$

where the value $S_{l}(\boldsymbol{r}, \omega)=S(\omega) P_{l}(\boldsymbol{r}, \omega)$ is called the spectral modal amplitude.

The first step in a modal analysis of the experimental data is to compare the depth dependence of the amplitudes (i.e., the modulus) of the theoretical and experimental modes. To obtain the depth dependence of the experimental modes, we use the difference between the group velocities of different modes $v_{l}^{g r}(\omega)$. In other words we cut a narrow frequency interval from the total band of the spectrum $S(\omega)$. This can be achieved by using integration with narrow band filtering function $F\left(\omega, \omega_{c}\right)$.

In the following analysis we will use a Gaussian form for $F\left(\omega, \omega_{c}\right)$, where $\omega_{c}$ is the middle of the frequency range of width $\Delta \omega, \Delta \omega \ll \omega_{c}$ :

$$
F\left(\omega, \omega_{c}\right)=\frac{1}{\Delta \omega \sqrt{2 \pi}} \exp \left\{-\frac{\left(\omega-\omega_{c}\right)^{2}}{2 \Delta \omega^{2}}\right\} .
$$

For a narrow frequency band, the time dependence of the received signal can be expressed as 
(a)
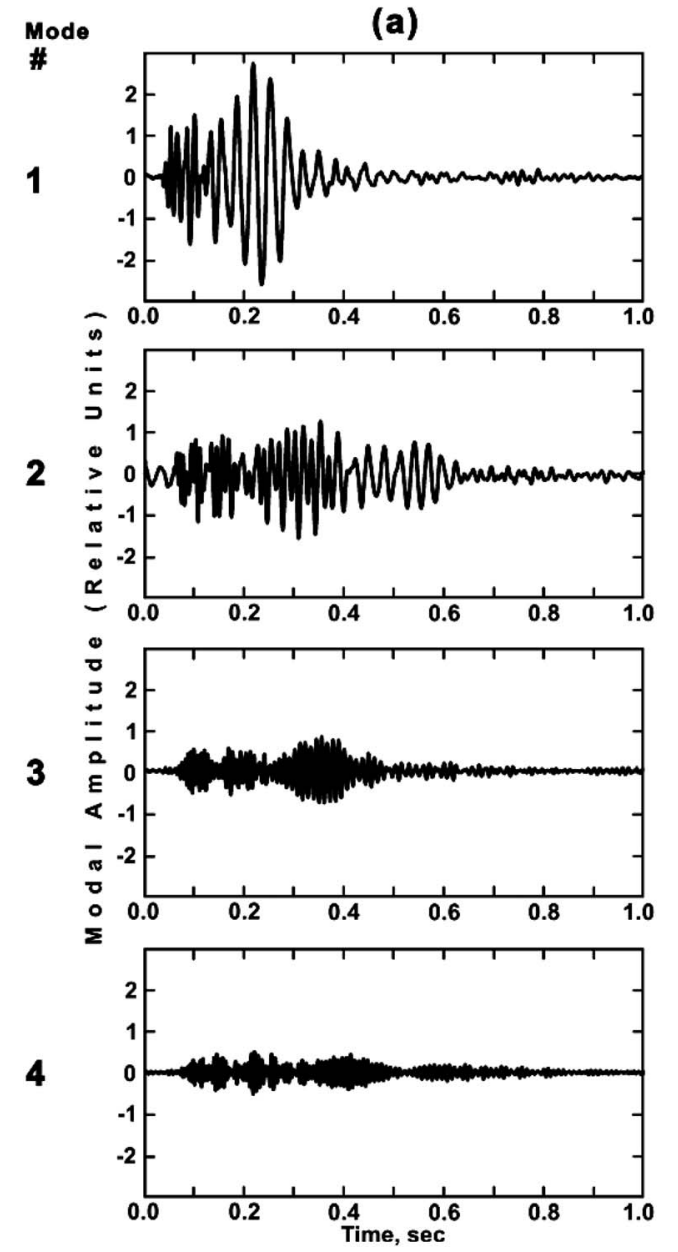

(b)
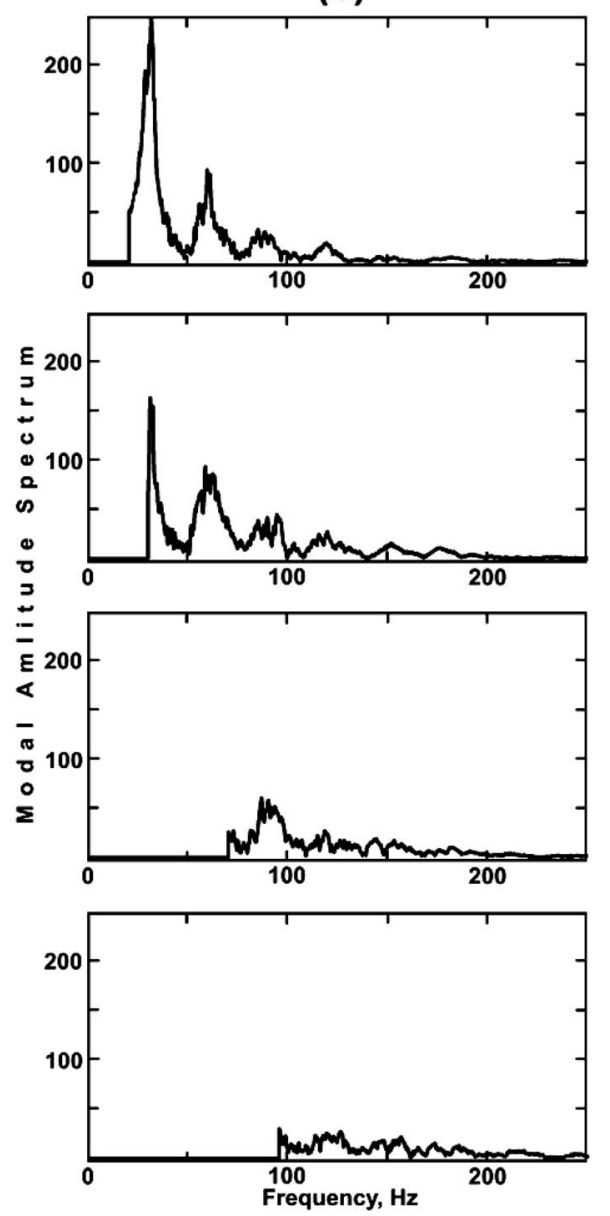

FIG. 13. Filtered modes 1-4 for $T=19: 15$ GMT. (a) Relative mode amplitude. (b) Modal amplitude.

$$
\widetilde{P}\left(\boldsymbol{r}, z, t, \omega_{c}\right)=2 \int_{0}^{\infty} F\left(\omega, \omega_{c}\right) S(\boldsymbol{r}, z, \omega) e^{-i \omega t} d \omega
$$

where the tilde $(\sim)$ denotes the filtered experimental values for fixed center frequency $\omega_{c}$. The results of the frequency filtering for $\Delta f=10 \mathrm{~Hz}$ (i.e., $\Delta \omega=2 \pi \Delta f$ ) for geotime $T$ $=19: 15 \mathrm{GMT}$ and $\omega_{c}=60,90 \mathrm{~Hz}$, respectively, for different hydrophones are presented in Fig. 10. The maxima in arrival times show the arrivals of different modes due to differences in group velocities, which can be used for mode filtering. We then split arrival times of pulses into intervals $\left(t_{l}, t_{l}+\Delta t\right)$, where $t_{l}=L / v_{l}^{g r}$ is the arrival time of mode $l$, and $\Delta t$ is the duration of the pulse. After integration of the pulse envelope (amplitude) we get a function of depth and frequency $\omega_{c}$, which is proportional to the amplitude of mode $l$, denoted as $\widetilde{\psi}_{l}\left(z, \omega_{c}\right)$ :

$$
\tilde{\psi}_{l}\left(z, \omega_{c}\right)=\int_{t_{l}}^{t_{l}+\Delta t}\left|\widetilde{p}\left(\boldsymbol{r}, z, t, \omega_{c}\right)\right| d t
$$

Consequently, we receive modulus of the first, second, and third modes, respectively.

Next we should compare the eigenfunctions obtained from the experimental data with the theoretically calculated values. For this we should normalize the selected modes using some relationship. To normalize experimental functions to compare with the analytical ones, we use

$$
\boldsymbol{\Psi}_{l}\left(z, \omega_{c}\right)=\tilde{\psi}_{l}\left(z, \omega_{c}\right) / \int_{0}^{H}\left[\tilde{\psi}_{l}\left(z, \omega_{c}\right)\right]^{2} d z .
$$

The results of mode filtering the data shown in Fig. 10(c) are presented in Fig. 11.

Excellent agreement between experimentally extracted and theoretically calculated modes is obtained; hence, for the next step in our analysis the calculated modes (i.e., the eigenfunctions of Sturm-Liouville theory) are used rather than filtering the experimental data as shown above.

Next the frequency dependence of modal amplitudes is obtained. Modes represented by $\psi_{l}(z, \omega)$ are orthogonal to each other and the integral over variable $z$ is extended from zero to infinity (including the sea bottom). However, the contribution of the sea bottom to the modal functions is small, particularly for the lower order modes. We can thus approximate

$$
\int_{0}^{H} \psi_{l}(z, \omega) \psi_{m}(z, \omega) d z \approx \delta_{n m}
$$

where $\delta_{m n}$ is the Kronecker delta function. According to (31) and (36), the spectral amplitude of the $l$ th mode can be ob- 

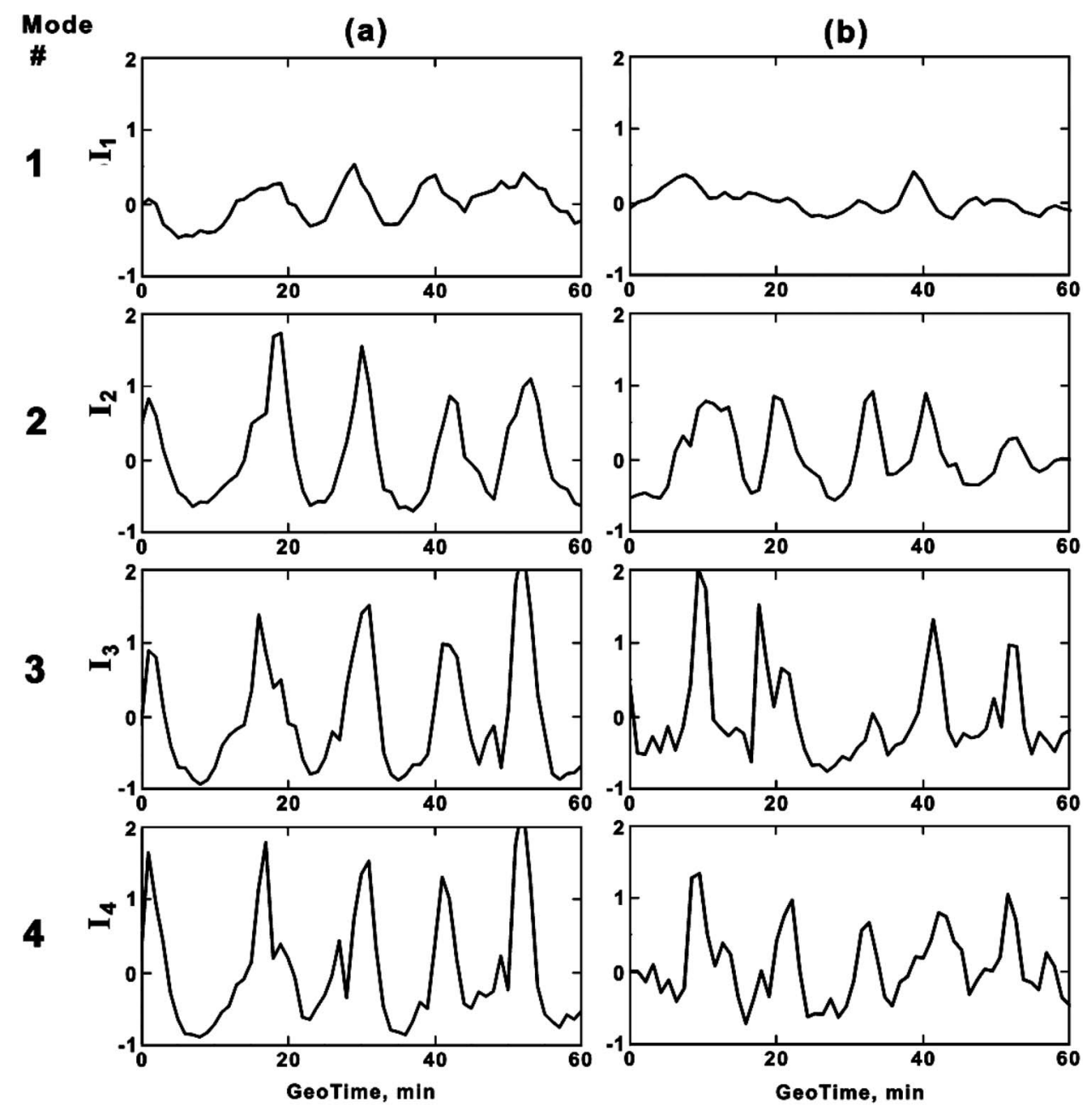

FIG. 14. Relative intensity fluctuations of modes 1-4 obtained from experimental data for (a) $T=19: 00-20: 00$ GMT and (b) $T=20: 00-21: 00$ GMT.

tained by integration of the spectrum of the received signal as a function of depth:

$$
S_{l}(\boldsymbol{r}, \omega)=\int_{0}^{H} S(\boldsymbol{r}, z, \omega) \psi_{l}(z, \omega) d z .
$$

The time-frequency diagram for different hydrophone depths of the vertical line array corresponds to the modal decomposition of filtered sound pressure (33) at the receiver:

$$
\begin{aligned}
& \widetilde{P}\left(\boldsymbol{r}, z, t, \omega_{c}\right)=\sum_{l} \psi_{l}(z) \tilde{P}_{l}\left(\boldsymbol{r} ; t, \omega_{c}\right), \\
& \widetilde{P}_{l}\left(\boldsymbol{r} ; t, \omega_{c}\right)=2 \int_{0}^{\infty} F\left(\omega, \omega_{c}\right) S_{l}(\boldsymbol{r}, \omega) e^{-i \omega t} d \omega .
\end{aligned}
$$

The value $\left|\widetilde{P}_{l}\left(\boldsymbol{r} ; t, \omega_{c}\right)\right|$ is shown in Fig. 12. The irregular shape of the time-frequency curves is the result of the source spectrum [see Figs. 6(b) and 6(d)]. These "hyperbolalike" curves coincide with the theoretically obtained values (not shown in this plot) calculated using the aforementioned model. Figure 13 shows the arrival time dependence of modal amplitudes resulting from mode filtering and the corresponding modal spectra for modes $1-4$. We note that the modal amplitude reduces as we go from mode 1 to 4 while the cutoff frequency increases. The initial (or radiated) am- plitudes of the separate modes are determined by the source position in the water depth. The modal intensity as a function of frequency $\omega$ for geotime $T$ is

$$
I_{l \omega}(T)=\frac{4 \pi}{\rho c}\left|S_{l}(\boldsymbol{r}, \omega)\right|^{2} .
$$

Dependence of modal intensity on geotime for fixed frequency (in this case $90 \mathrm{~Hz}$ ) is shown in Fig. 14; similar curves have been constructed for another frequency in the frequency band of our sources, not shown here. For each curve we can calculate the value of fluctuations for a given geotime interval (e.g., $2 \mathrm{~h}$ here) and thus the corresponding scintillation index. The frequency dependence of modal intensity fluctuations through the scintillation index (29) can be obtained, and for this data are shown in Fig. 15. These dependencies, as we can see from Fig. 15, have irregular behavior as a function of frequency. Thus we will consider the smoothed dependence obtained by

$$
S I_{l}^{2}(\omega)=\frac{\left\langle\delta I^{2}\left(\omega, T_{i}\right)\right\rangle}{\left\langle I\left(\omega, T_{i}\right)\right\rangle^{2}},
$$

where \langle\rangle denotes an averaged value in time. Finally, in Fig. 16 we present these smoothed curves of the scintillation index for two independent geotime periods, for cases 1 and 2 . 

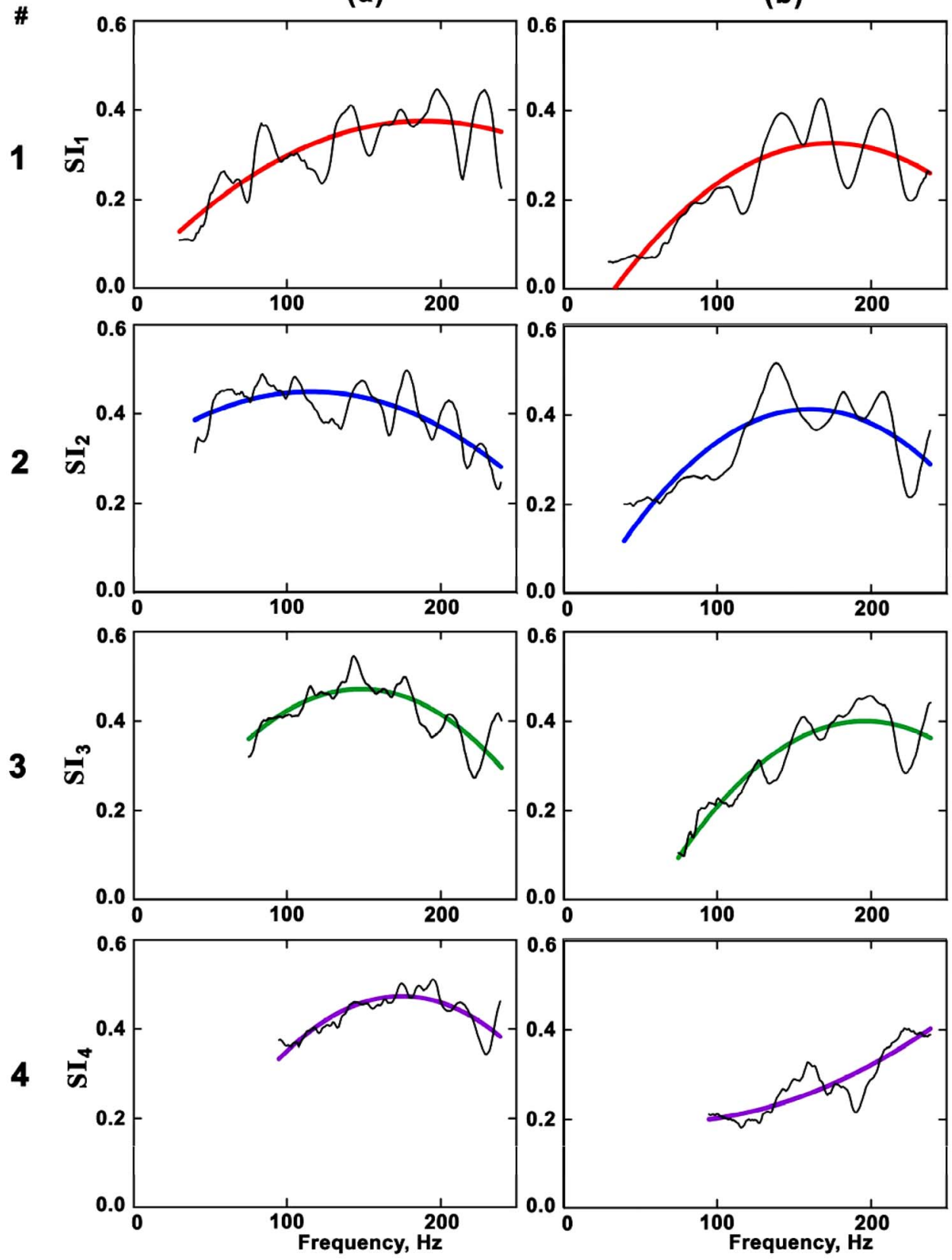

FIG. 15. (Color online) Frequency dependent modal scintillation index, SI $(f)$ of modes 1-4 obtained from experimental data for (a) $T=19: 00-20: 00$ GMT and (b) $T=20: 00-21: 00$ GMT. The data are shown by oscillating curves and averaged values (smooth lines).

\section{SUMMARY AND CONCLUSIONS}

A theory of sound propagation through an anisotropic shallow water environment is presented to examine the frequency dependence of the scintillation index in shallow water in the presence of internal waves. The theory of horizontal rays and vertical modes is used to establish the azimuthal behavior of the intensity fluctuations of the broadband acoustic signals propagating through shallow water internal waves.

In an earlier paper $^{6}$ we have shown that the temporal variations of depth dependence of intensity of the sound field resulted from horizontal anisotropy (referred to as the violation of circular symmetry) taking place in the presence of solitary internal waves. In addition, we noted the similarity of frequency dependence of modal scintillation index (for temporal fluctuations of intensity) and the index of horizontal refraction of the corresponding modes. We hypothesized that there is a link between the two.

In this paper we have extended our consideration of the nature of fluctuations of the sound field in directional dependence of propagation relative to the wave front of the ISW. Depending on the angle between the direction of the internal wave front and the acoustic track, the mechanism of sound field fluctuations is characterized by either horizontal refraction (HR) or horizontal focusing (HF), is adiabatic (AD), or is a mode coupling mechanism (MC) as shown in Fig. 1.

In this paper we have presented a theory and corresponding experimental results for the sector HR in Fig. 1, where fluctuations are caused by the effect of horizontal re- 
(a)

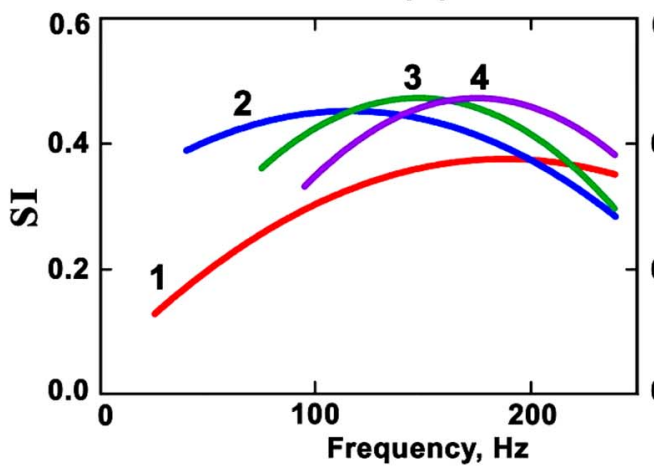

(b)

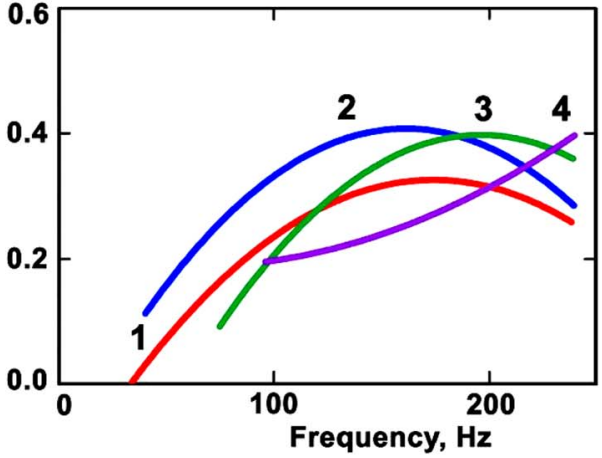

FIG. 16. (Color online) Frequency dependence of modal scintillation index SI ( $f$ ) for (a) $T=19: 00-20: 00$ GMT and (b) $T=20: 00-21: 00$ GMT.

fraction or (more generally) by redistribution of the sound field in the horizontal plane. Using a ray approximation in the horizontal plane, in this paper we derived an analytical expression for the scintillation index of a sound field due to moving internal waves in shallow water.

The main feature of the frequency dependence of the modal scintillation index (29) is the shape of this dependence shown in Fig. 16. This figure, which is obtained from the experimental data and corresponding to theoretical Fig. 3, shows the relationship between the modal scintillation index, SI, and the parameters of the waveguide. We further see that this does not depend on the amplitude and shape of the internal waves.

Note that factor $\zeta_{0} / \sin ^{2} \chi_{s}$ has a constant value for a given sequence of sound pulses. In this connection the value of the horizontal refraction index plays the role of an invariant characteristic of a definite geographical shallow water area, and one that determines the fluctuations of the sound field in the corresponding conditions (i.e., HR sector in horizontal plane).

A good agreement between the experimental and analytical results in this paper, in spite of the idealized modeling of the internal waves, is a confirmation of the fact that the scintillation index (SI) is independent of (or weakly dependent on) the parameters of the internal waves such as amplitude, period, and wave number. We note further that the comparison between the experimental and theoretical values is very good for the first hour [Fig. 16(a)] when there is a distinct internal wave passing through, while it is less good for the second hour [Fig. 16(b)], where the amplitude of the internal waves has considerably reduced. Hence, the anisotropic properties of the shallow water channel are less affected by the more isotropic background internal waves.

\section{ACKNOWLEDGMENTS}

This work was supported by the Ocean Acoustics Program (321 OA) of the Office of Naval Research (ONR Grant
No. N00014-01-1-0114 to UD and N00014-04-10146 to WHOI) and by the Russian Foundation for Basic Research (RFBR Grant No. 06-05-64853-a). Funding from Delaware Sea Grant program was provided to support graduate students at the University of Delaware.

${ }^{1}$ J. R. Apel, M. Badiey, C.-S. Chiu, S. Finette, R. H. Headrick, J. Kemp, J. F. Lynch, A. E. Newhall, M. H. Orr, B. H. Pasewark, D. Tielburger, A. Turgut, K. von der Heydt, and S. N. Wolf, "An overview of the SWARM 1995 shallow-water internal wave acoustic scattering experiment," IEEE J. Ocean. Eng. 22, 465-500 (1997).

${ }^{2}$ M. Badiey, Y. Mu, J. F. Lynch, J. R. Apel, and S. N. Wolf, "Temporal and azimuthal dependence of sound propagation in shallow water with internal waves," IEEE J. Ocean. Eng. 27, 117-129 (2002).

${ }^{3}$ R. H. Headrick, J. F. Lynch, Acoustic normal mode fluctuation statistics in the 1995 SWARM internal wave scattering experiment.," J. Acoust. Soc. Am. 107, 201-220; and the SWARM group, "Modeling mode arrivals in the 1995 SWARM experiment acoustic transmissions," J. Acoust. Soc. Am. 107, 220-236 (2000).

${ }^{4}$ D. Rubenstein and M. N. Brill, "Acoustic variability due to internal waves and surface waves in shallow water," in Ocean Variability and Acoustics Propagation, edited by J. Potter and A. Warn-Varnas (Kluwer Academic, Dordrecht, 1991), pp. 215-228.

${ }^{5}$ D. Rubenstein, "Observations of cnoidal internal waves and their effect on acoustic propagation in shallow water," IEEE J. Ocean. Eng. 24, 346-357 (1999).

${ }^{6}$ M. Badiey, B. Katsnelson, J. Lynch, S. Pereselkov, and W. Siegmann, "Measurement and modeling of 3-D sound intensity variations due to shallow water internal waves," J. Acoust. Soc. Am. 117, 613-625 (2005).

${ }^{7}$ H. Weinberg and R. Burridge, "Horizontal ray theory for ocean acoustics," J. Acoust. Soc. Am. 55, 63-79 (1974).

${ }^{8}$ S. D. Frank, M. Badiey, J. F. Lynch, and W. L. Siegmann, "Analysis and modeling of broadband airgun data influenced by nonlinear internal waves," J. Acoust. Soc. Am. 116(6), 3404-3422 (2004).

${ }^{9}$ S. D. Frank, M. Badiey, and W. L. Siegmann, "Experimental evidence of three-diemensional acoustic propagation caused by nonlinear internal waves," J. Acoust. Soc. Am. 118(2), 723-734 (2005).

${ }^{10}$ B. G. Katsnelson and S. A. Pereselkov, "Low-frequency horizontal acoustic refraction caused by internal wave solitons in a shallow sea," Acoust. Phys. 46, 684-691 (2000).

${ }^{11}$ B. G. Katsnelson and S. A. Pereselkov, "Space-frequency dependence of the horizontal structure of the sound field in the presence of intense internal waves," Acoust. Phys. 50, 169-176 (2004).

${ }^{12}$ A. Ishimaru, Wave Propagation and Scattering in Random Media (Academic, New York, 1978), Vol. 2. 